\title{
On Trace-Convex Noncommutative Polynomials
}

\author{
Igor Klep, Scott A. McCullough, \\ \& CHRISTOPHER S. NELSON
}

Dedicated to Bill Helton on the occasion of his ?? birthday

\begin{abstract}
To each continuous function $f: \mathbb{R} \rightarrow \mathbb{R}$ there is an associated trace function on $n \times n$ real symmetric matrices $\operatorname{Tr} f$. The classical Klein lemma states that $f$ is convex if and only if $\operatorname{Tr} f$ is convex. In this note we present an algebraic strengthening of this lemma for univariate polynomials $f: \operatorname{Tr} f$ is convex if and only if the noncommutative second directional derivative of $f$ is a sum of Hermitian squares and commutators in a free algebra. We also give a localized version of this result.
\end{abstract}

\section{Introduction}

Trace-convexity is a notion frequently used in free probability and free analysis [SV06; KV14], where, for example, the trace of certain potentials is assumed to be convex; see [Gui06; Car10; GS09] or the references therein. One of the basic technical tools of the trade is the so-called Klein lemma saying that a continuous function $f: \mathbb{R} \rightarrow \mathbb{R}$ is convex if and only if the associated trace function $\operatorname{Tr} f$ : $\mathbb{S}_{n} \rightarrow \mathbb{R}$ is convex for all $n \in \mathbb{N}$. (Here $\mathbb{S}_{n}$ denotes the set of all real symmetric $n \times n$ matrices.) We call such a function trace-convex.

In this note we establish an algebraic version of Klein's lemma. That is, we give an algebraic certificate using sums of squares and commutators in the free algebra on two letters whose existence is equivalent to trace-convexity of a polynomial. Indeed, we show that trace-convexity of a univariate real polynomial $p$ is equivalent to its second noncommutative derivative $p^{\prime \prime}(x)[h]$ being a sum of Hermitian squares plus commutators.

The article is organized as follows. Section 2 fixes notation, terminology, and gives some preliminaries. Then Section 3 contains our main results, and we conclude with remarks and algorithmic considerations in Section 4.

Received December 19, 2014. Revision received October 27, 2015.

The first author is supported by the Marsden Fund Council of the Royal Society of New Zealand. Partially supported by the Slovenian Research Agency grants P1-0222, L1-4292 and L1-6722. Part of this research was done while the author was on leave from the University of Maribor. The second author's research is supported by the NSF grants DMS 1101137 and 1361501. The third author is partly supported by the National Science Foundation, DMS 1201498. 


\section{Notation and Preliminaries}

\subsection{Matrices}

There is a natural partial ordering on $\mathbb{S}_{n}$ defined by $X \succeq Y$ if the symmetric matrix $X-Y$ is positive semidefinite, that is, if its eigenvalues are all nonnegative. Similarly, $X \succ Y$ if $X-Y$ is positive definite, that is, all its eigenvalues are positive.

\subsection{Noncommutative (nc) Polynomials}

Even if $p$ is a univariate polynomial, it naturally has noncommutative derivatives, which are polynomials in two freely noncommuting variables.

Let $x=\left(x_{1}, \ldots, x_{g}\right)$ denote a $g$-tuple of free noncommuting variables, and let $\mathbb{R}\langle x\rangle$ denote the associative $\mathbb{R}$-algebra freely generated by $x$. Its elements are called (nc) polynomials. An element of the form $a w$ where $0 \neq a \in \mathbb{R}$ and $w$ is a word in the variables $x$ is called a monomial and $a$ its coefficient. The empty word $\varnothing$ is the multiplicative identity for $\mathbb{R}\langle x\rangle$.

There is a natural involution ${ }^{T}$ on $\mathbb{R}\langle x\rangle$ that reverses words. For example,

$$
\left(2-3 x_{1}^{2} x_{2} x_{3}\right)^{T}=2-3 x_{3} x_{2} x_{1}^{2} .
$$

A polynomial $p$ is a symmetric polynomial if $p^{T}=p$. Because $x_{j}^{T}=x_{j}$, we refer to the variables as symmetric variables. The degree of an nc polynomial $p$, denoted $\operatorname{deg}(p)$, is the length of the longest word appearing in $p$. Let $\mathbb{R}\langle x\rangle_{k}$ denote the polynomials of degree at most $k$.

\subsection{Derivatives}

Given a polynomial $p \in \mathbb{R}\langle x\rangle$, the $\ell$ th noncommutative directional derivative of $p$ in the direction $h$ is

$$
p^{(\ell)}(x)[h]:=\left.\frac{d^{\ell} p(x+t h)}{d t^{\ell}}\right|_{t=0} .
$$

Thus, $p^{(\ell)}(x)[h]$ is the polynomial that evaluates to

$$
\left.\frac{d^{\ell} p(X+t H)}{d t^{\ell}}\right|_{t=0} \text { for every } n \in \mathbb{N} \text { and every choice of tuples } X, H \in \mathbb{S}_{n}^{g} \text {. }
$$

Let $p^{\prime}(x)[h]$ denote the noncommutative first derivative of $p$ and we denote the Hessian, the second noncommutative derivative of $p$ in the direction $h$, by $p^{\prime \prime}(x)[h]$. Equivalently, the Hessian of $p$ can also be defined as the part of the noncommutative polynomial

$$
r(x)[h]:=2(p(x+h)-p(x)) \in \mathbb{R}\langle x\rangle[h]:=\mathbb{R}\left\langle x_{1}, \ldots, x_{g}, h_{1}, \ldots, h_{g}\right\rangle
$$

that is homogeneous of degree two in $h$.

If $p^{\prime \prime} \neq 0$, that is, if $p$ is an nc polynomial of degree two or more, then its Hessian $p^{\prime \prime}(x)[h]$ is a polynomial in the $2 g$ variables $x_{1}, \ldots, x_{g}, h_{1} \ldots, h_{g}$, which is homogeneous of degree two in $h$ and has degree equal to the degree of $p$. 


\subsection{Commutators, Cyclic Equivalence}

A polynomial of the form $[p, q]:=p q-q p$ for $p, q \in \mathbb{R}\langle x\rangle$ is a commutator. Two polynomials $f, g \in \mathbb{R}\langle x\rangle$ are called cyclically equivalent $(f \stackrel{\text { cyc }}{\sim} g$ ) if $f-g$ is a sum of commutators in $\mathbb{R}\langle x\rangle$. Cyclic equivalence can be easily checked; see [KS08, Remark 1.3].

\subsection{Trace-Convexity}

We now introduce the central notion used in this article.

Definition 2.1. A symmetric polynomial $p \in \mathbb{R}\langle x\rangle$ is trace-convex if for each $n$ and each pair of $g$ tuples of $n \times n$ symmetric matrices $X=\left(X_{1}, \ldots, X_{g}\right)$ and $Y=\left(Y_{1}, \ldots, Y_{g}\right)$, we have

$$
\frac{1}{2}(\operatorname{Tr} p(X)+\operatorname{Tr} p(Y)) \geq \operatorname{Tr} p\left(\frac{X+Y}{2}\right) .
$$

Equivalently,

$$
\frac{\operatorname{Tr} p(X)+\operatorname{Tr} p(Y)}{2}-\operatorname{Tr} p\left(\frac{X+Y}{2}\right) \geq 0 .
$$

We sometimes restrict (2.1) or, equivalently, (2.2), to hold only for $X, Y$ in a domain $\mathcal{D}$. In this case, $p$ is trace-convex on $\mathcal{D}$.

\subsection{Related Notions}

Noncommutative polynomials and noncommutative rational functions arise in several contexts including systems theory [BGM06; BGM05] and is a part of the new field of free (freely noncommutative) analysis [SV06; KV14; AM15]. Sums of squares representations are a theme in both the commutative and noncommutative settings [Pu13].

There is a related notion of matrix-convexity of an nc polynomial. A symmetric polynomial $p \in \mathbb{R}\langle x\rangle$ is matrix-convex if

$$
\frac{p(X)+p(Y)}{2}-p\left(\frac{X+Y}{2}\right) \succeq 0
$$

for all tuples of symmetric matrices $X, Y$. Note, that even if $p$ is a univariate polynomial, $p(x+y)$ is an nc polynomial in $x, y$ since $X$ and $Y$ need not commute. This convexity condition is very strong and leads to extreme rigidity. For instance, by Helton and McCullough [HM04a], every matrix-convex polynomial is of degree at most two, a result that depends on two key observations. First, $p$ is matrix-convex if and only if $p^{\prime \prime}(x)[h]$ is matrix-positive, that is,

$$
p^{\prime \prime}(X)[H] \succeq 0
$$


for all $X, H \in \mathbb{S}_{n}^{g}$ and $n \in \mathbb{N}$ [CHSY03]. Second, $q \in \mathbb{R}\langle x\rangle$ is matrix-positive if and only if it is a sum of Hermitian squares, that is,

$$
q=\sum_{j} r_{j}^{T} r_{j}
$$

for some $r_{j} \in \mathbb{R}\langle x\rangle$ [Hel02; McC01].

We refer the reader to [Kra36; Eff09; Han97; OST07; Uci02] for further studies of operator-monotonicity.

\section{Results}

We are now ready to present the main results of this article characterizing univariate trace-convex polynomials with algebraic certificates involving sums of squares.

THeOREM 3.1 (global version). If $p$ is a univariate polynomial, then the following are equivalent.

(i) $p$ is convex;

(ii) $\operatorname{Tr} p$ is convex, that is, $p$ is trace-convex;

(iii) $p^{\prime \prime}(x)[h]$ is a sum of Hermitian squares and commutators.

Next, we present a local version of Theorem 3.1 characterizing univariate polynomials that are trace-convex on a matrix-interval. This time the algebraic certificates involve weighted sums of squares.

TheOREM 3.2 (local version). Suppose $p$ is a univariate polynomial and $-\infty<$ $a<b<\infty$.

(1) $\operatorname{Tr} p$ is convex on $a I \prec X \prec b I$ if and only if $p$ is convex on $(a, b)$ if and only if $p^{\prime \prime}(x)[h]$ is cyclically equivalent to a polynomial of the form

$$
\begin{aligned}
& \sum_{i}^{\text {finite }} q_{i}(x)[h]^{T} q_{i}(x)[h]+\sum_{i}^{\text {finite }} r_{i}(x)[h]^{T}(x-a) r_{i}(x)[h] \\
& \quad+\sum_{i}^{\text {finite }} s_{i}(x)[h]^{T}(b-x) s_{i}(x)[h]+\sum_{i}^{\text {finite }} t_{i}(x)[h]^{T}(x-a)(b-x) t_{i}(x)[h] \\
& \quad+\sum_{i}^{\text {finite }}(x-a) u_{i}(x)[h]^{T}(b-x) u_{i}(x)[h]
\end{aligned}
$$

for some $q_{i}, r_{i}, s_{i}, t_{i}, u_{i} \in \mathbb{R}\langle x\rangle[h]$ homogeneous of degree one in $h$.

(2) $\operatorname{Tr} p$ is convex on $b I \prec X$ if and only if $p$ is convex on $(b, \infty)$ if and only if $p^{\prime \prime}(x)[h]$ is cyclically equivalent to a polynomial of the form

$$
\sum_{i}^{\text {finite }} q_{i}(x)[h]^{T} q_{i}(x)[h]+\sum_{j}^{\text {finite }} r_{j}(x)[h]^{T}(x-b) r_{j}(x)[h] .
$$


(3) $\operatorname{Tr} p$ is convex on $X \prec a I$ if and only if $p$ is convex on $(-\infty, a)$ if and only if $p^{\prime \prime}(x)[h]$ is cyclically equivalent to a polynomial of the form

$$
\sum_{i}^{\text {finite }} q_{i}(x)[h]^{T} q_{i}(x)[h]+\sum_{j}^{\text {finite }} r_{j}(x)[h]^{T}(a-x) r_{j}(x)[h] .
$$

The proofs of Theorems 3.1 and 3.2 occupy the remainder of this section.

\subsection{Symmetrizer}

One of our main tools in analyzing second derivatives of univariate nc polynomials is the following operation of symmetrization.

Definition 3.3. Given $d \in \mathbb{N}$ and nc polynomials $y_{1}, \ldots, y_{d}$, define $\operatorname{Sym}_{d}\left(y_{1}\right.$, $\ldots, y_{d}$ ) to be the nc polynomial

$$
\operatorname{Sym}_{d}\left(y_{1}, \ldots, y_{d}\right)=\frac{1}{d !} \sum_{\sigma \in S_{d}} y_{\sigma(1)} \cdots y_{\sigma(d)} .
$$

Often we will omit the subscript $d$.

LemMa 3.4. Let $y_{1}, \ldots, y_{d}$ be nc polynomials. If $\sigma \in S_{d}$, then

$$
\operatorname{Sym}_{d}\left(y_{\sigma(1)}, \ldots, y_{\sigma(d)}\right)=\operatorname{Sym}_{d}\left(y_{1}, \ldots, y_{d}\right) .
$$

Proof. Trivial.

Lemma 3.5. Fix $1 \leq k \leq d$. If $y_{1}, \ldots, y_{d}$ are $n c$ polynomials and $a_{k}$ is a constant, then

$$
\begin{aligned}
\operatorname{Sym}_{d} & \left(y_{1}, \ldots, y_{k-1}, y_{k}-a_{k}, y_{k+1}, \ldots, y_{d}\right) \\
= & \operatorname{Sym}_{d}\left(y_{1}, \ldots, y_{k-1}, y_{k}, y_{k+1}, \ldots, y_{d}\right) \\
& \quad-a_{k} \operatorname{Sym}_{d-1}\left(y_{1}, \ldots, y_{k-1}, y_{k+1}, \ldots, y_{d}\right) .
\end{aligned}
$$

Proof. The polynomial $\operatorname{Sym}_{d}\left(y_{1}, \ldots, y_{k-1}, y_{k}-a_{k}, y_{k+1}, \ldots, y_{d}\right)$ is a sum of products of polynomials, each of which contains $y_{k}-a_{k}$. Distributing the $y_{k}-a_{k}$ in each product gives

$$
\frac{1}{d !} \sum_{\sigma \in S_{d}} y_{\sigma(1)} \cdots y_{\sigma(d)}-a_{k} \frac{1}{d !} \sum_{j=1}^{d} \sum_{\sigma(j)=k} y_{\sigma(1)} \cdots y_{\sigma(j-1)} y_{\sigma(j+1)} \cdots y_{\sigma(d)} \text {. }
$$

For $1 \leq j \leq d$, let $S_{d, j}$ denote those $\sigma \in S_{d}$ such that $\sigma(j)=k$. Given a permutation $\sigma \in S_{d, j}$, define $\tilde{\sigma}$ to be

$$
\tilde{\sigma}(i)= \begin{cases}k, & i=1, \\ \sigma(i-1), & 1 \leq i-1<j, \\ \sigma(i), & j<i .\end{cases}
$$


Given a fixed $j$, the mapping from $S_{d, j}$ to $S_{d, 1}$ defined by $\sigma \mapsto \tilde{\sigma}$ is a bijection, and further,

$$
y_{\tilde{\sigma}(2)} \cdots y_{\tilde{\sigma}(d)}=y_{\sigma(1)} \cdots y_{\sigma(j-1)} y_{\sigma(j+1)} \cdots y_{\sigma(d)} .
$$

Therefore, (3.2) simplifies to

$$
\begin{gathered}
\frac{1}{d !} \sum_{\sigma \in S_{d}} y_{\sigma(1)} \cdots y_{\sigma(d)}-a_{k} \frac{1}{(d-1) !} \sum_{\sigma(1)=k} y_{\sigma(2)} \cdots y_{\sigma(d)} \\
=\operatorname{Sym}_{d}\left(y_{1}, \ldots, y_{k-1}, y_{k}, y_{k+1}, \ldots, y_{d}\right) \\
\quad-a_{k} \operatorname{Sym}_{d-1}\left(y_{1}, \ldots, y_{k-1}, y_{k+1}, \ldots, y_{d}\right) .
\end{gathered}
$$

\subsection{From Commutative to Noncommutative Polynomials}

For a univariate polynomial $p$, let $\frac{d p}{d x}$ and $\frac{d^{2} p}{d x^{2}}$ denote the ordinary first and second derivative of $p$ (and $p^{\prime}$ and $p^{\prime \prime}$ the first and second nc derivative of $p$ ).

LEMMA 3.6. Let $p$ be a univariate polynomial. If

$$
\frac{d^{2} p}{d x^{2}}=\left(x-a_{1}\right) \cdots\left(x-a_{d}\right)
$$

then

$$
p^{\prime \prime}(x)[h]=\operatorname{Sym}_{d+2}\left(x-a_{1}, \ldots, x-a_{d}, h, h\right) .
$$

Proof. First, consider the simple case $\frac{d^{2} p}{d x^{2}}(x)=x^{d}$ for which

$$
p=\frac{1}{(d+2)(d+1)} x^{d+2}+\ell,
$$

where $\ell$ is some linear polynomial. Computing the second nc derivative of $x^{d+2}$ gives two times the sum of all words of degree $d$ in $x$ and degree two in $h$. Therefore, for each $i<j$, the coefficient of the word with an $h$ as the $i$ th and $j$ th letters in $p^{\prime \prime}(x)[h]$ is

$$
\frac{2}{(d+2)(d+1)} \text {. }
$$

Examining $\operatorname{Sym}(x, \ldots, x, h, h)$, we see that, for each $i<j$, the coefficient of the word with an $h$ as the $i$ th and $j$ th letters is

$$
\frac{1}{(d+2) !} d ! 2 !=\frac{2}{(d+2)(d+1)} .
$$

Next, consider the general case $\frac{d^{2} p}{d x^{2}}=\left(x-a_{1}\right) \ldots\left(x-a_{d}\right)$. We see that

$$
\frac{d^{2} p}{d x^{2}}=\sum_{k=0}^{d}\left(\sum_{1 \leq i_{1}<\cdots<i_{d-k} \leq n}(-1)^{d-k} a_{i_{1}} \ldots a_{i_{d-k}} x^{k}\right) .
$$


By linearity,

$$
p^{\prime \prime}(x)[h]=\sum_{k=0}^{d} \sum_{1 \leq i_{1}<\cdots<i_{d-k} \leq n}(-1)^{d-k} a_{i_{1}} \ldots a_{i_{d-k}} \operatorname{Sym}_{k+2}(x, \ldots, x, h, h) .
$$

Repeated application of Lemma 3.5 to each $\left(x-a_{i}\right)$ in the expression $\operatorname{Sym}(x-$ $\left.a_{1}, \ldots, x-a_{d}, h, h\right)$ shows that this last expression is equal to $\operatorname{Sym}(x-$ $\left.a_{1}, \ldots, x-a_{d}, h, h\right)$.

\subsection{On Hankel Matrices}

Recall that an $(n+1) \times(n+1)$ square matrix $T$ is called Hankel if it has constant antidiagonals, that is,

$$
T_{i, j}=T_{i-1, j+1}
$$

for $0<i \leq n$ and $0 \leq j<n$. A finite sequence $c_{0}, \ldots, c_{2 m}$ generates an $(m+1) \times$ $(m+1)$ Hankel matrix $T$ with

$$
T_{i, j}=c_{i+j}, \quad 0 \leq i, j \leq m .
$$

We refer to [Dym07] for more on Hankel matrices.

Lemma 3.7. For all $k, \ell, d \in \mathbb{N}$ with $\ell \leq k$, there is a regular Borel measure supported on $\mathbb{R}$ such that

$$
\frac{1}{\left(\begin{array}{c}
2 d+k \\
j+\ell
\end{array}\right)}=\int x^{j} d \mu
$$

for $0 \leq j \leq 2 d$. In particular, the Hankel matrix generated by the finite sequence $\left(1 /\left(\begin{array}{c}2 d+k \\ \ell+j\end{array}\right)\right)_{j=0}^{2 d}$ is positive semidefinite.

Proof. Consider the measure

$$
d \mu=\frac{2 d+k+1}{(x+1)^{2 d+k+2}} x^{k} d x
$$

on the half-line $[0, \infty)$. The $j$ th moment of this measure is

$$
\int_{0}^{\infty} x^{j+k} \frac{2 d+k+1}{(x+1)^{2 d+k+2}} d x \text {. }
$$

Using the substitution $x=\frac{1}{t}-1$ gives

$$
\begin{aligned}
& \int_{0}^{1}(2 d+k+1)(1-t)^{j+k} t^{2 d+k-(j+k)} d t \\
& \quad=(2 d+k+1) B(2 d+k-(j+k)+1,(j+k)+1)=\frac{1}{\left(\begin{array}{c}
2 d+k \\
j+k
\end{array}\right)},
\end{aligned}
$$

where $B(x, y)$ denotes the beta function. 
Lemma 3.8. Given a positive integer $d$ and $n_{0}, n_{1}, \ldots, n_{d} \in \mathbb{N}$, let $\mathbf{1}_{i, j}$ denote the $n_{i} \times n_{j}$ matrix all of whose entries are 1 . If $H=\left(H_{i, j}\right)_{i, j=0}^{d}$ is a positive semidefinite $(d+1) \times(d+1)$ matrix, then the block matrix (of total size $n \times n$ where $\left.n=\sum n_{j}\right)$

is also positive semidefinite.

$$
C=\left(H_{i, j} \mathbf{1}_{i, j}\right)_{i, j=0}^{d}
$$

Proof. Let $N$ denote the max of $\left\{n_{0}, \ldots, n_{d}\right\}$, and let 1 denote the $N \times N$ matrix each of whose entries is 1 . The tensor (Kronecker) product $H \otimes \mathbf{1}$ is positive semidefinite since both $H$ and $\mathbf{1}$ are. Finally, $C$ is obtained from $H \otimes \mathbf{1}$ by compressing to a subspace (removing appropriate rows and columns) and is thus positive semidefinite.

\subsection{A Uniform Representation of the Symmetrizer}

Proposition 3.9. Fix a positive integer $d$ and nonnegative integer $k$. There exists a vector-valued polynomial $W(x, h, c)$ in the $n c$ variables $x$ and $h$ and commuting variables $c=\left(c_{1}, \ldots, c_{d}\right)$ (thus, each $c_{j}$ commutes with all other variables) and positive semidefinite matrices $C$ and $C_{0}, \ldots, C_{k}$ such that:

(1) the polynomial $\operatorname{Sym}\left(x-b_{1}, \ldots, x-b_{2 d}, h_{1}, h_{2}\right)$ (in the nc variables $x, h_{1}$, $h_{2}$ and commuting variables $\left.b_{1}, \ldots, b_{2 d}\right)$ is cyclically equivalent to

$$
W\left(x, h_{1}, b_{1}, \ldots, b_{d}\right)^{T} C W\left(x, h_{2}, b_{d+1}, \ldots, b_{2 d}\right) ;
$$

(2) the polynomial $\operatorname{Sym}\left(x-a_{1}, \ldots, x-a_{k}, x-b_{1}, \ldots, x-b_{2 d}, h_{1}, h_{2}\right)$ (in the $n c$ variables $x, h_{1}, h_{2}$ and commuting variables $\left.a_{1}, \ldots, a_{k} ; b_{1}, \ldots, b_{2 d}\right)$ is cyclically equivalent to

$$
\begin{gathered}
\sum_{\ell=0}^{k} \sum_{\tau \in S_{k}}\left(x-a_{\tau(1)}\right) \cdots\left(x-a_{\tau(\ell)}\right) W\left(x, h_{1}, b_{1}, \ldots, b_{d}\right)^{T}\left(x-a_{\tau(\ell+1)}\right) \\
\cdots\left(x-a_{\tau(k)}\right) C_{\ell} W\left(x, h_{2}, b_{d+1}, \ldots, b_{2 d}\right) .
\end{gathered}
$$

Proof. Note that equation (3.3) follows from equation (3.4) by choosing $k=0$. The polynomial $\operatorname{Sym}\left(x-a_{1}, \ldots, x-a_{k}, x-b_{1}, \ldots, x-b_{2 d}, h_{1}, h_{2}\right)$ is a sum of products that can be cyclically permuted so that they are of the form

$$
\begin{gathered}
\left(x-a_{\tau(1)}\right) \cdots\left(x-a_{\tau(\ell)}\right)\left(x-b_{\sigma(1)}\right) \cdots\left(x-b_{\sigma(m)}\right) h_{1}\left(x-a_{\tau(\ell+1)}\right) \\
\cdots\left(x-a_{\tau(k)}\right)\left(x-b_{\sigma(m+1)}\right) \cdots\left(x-b_{\sigma(2 d)}\right) h_{2},
\end{gathered}
$$

for some $0 \leq \ell \leq k, 0 \leq m \leq 2 d, \sigma \in S_{2 d}, \tau \in S_{k}$. There are $2 d+k+2$ cyclic permutations of (3.5) within $\operatorname{Sym}\left(x-a_{1}, \ldots, x-a_{k}, x-b_{1}, \ldots, x-b_{2 d}, h_{1}, h_{2}\right)$. Further, the factors of the form $x-c$, with $c$ a scalar, can be commuted with each other as long as they do not pass over an $h_{j}$. Given a set of factors that appear to the left of $h_{1}$, the other side of $h_{1}$ must consist of the remaining unused factors. This means that up to cyclic permutation each term is uniquely determined by which factors appear between $h_{1}$ and $h_{2}$. Therefore, the polynomial $\operatorname{Sym}(x-$ 
$\left.a_{1}, \ldots, x-a_{k}, x-b_{1}, \ldots, x-b_{2 d}, h_{1}, h_{2}\right)$, up to cyclic equivalence, contains a product of the form (3.5) with coefficient equal to

$$
\frac{(\ell+m) !(2 d+k-\ell-m) !(2 d+k+2)}{(2 d+k+2) !}=\frac{1}{\left(\begin{array}{c}
2 d+k \\
\ell+m
\end{array}\right)(2 d+k+1)} .
$$

By cyclic permutation, we can express (3.5) uniquely in the form

$$
\begin{aligned}
(x- & \left.a_{\tau(1)}\right) \cdots\left(x-a_{\tau(\ell)}\right)\left(x-b_{\phi(1)}\right) \cdots\left(x-b_{\phi(d-r)}\right) h_{1}\left(x-b_{\phi(d-r+1)}\right) \\
& \cdots\left(x-b_{\phi(d)}\right)\left(x-a_{\tau(\ell+1)}\right) \cdots\left(x-a_{\tau(d)}\right)\left(x-b_{d+\rho(1)}\right) \\
& \cdots\left(x-b_{d+\rho(s)}\right) h_{2}\left(x-b_{d+\rho(s+1)}\right) \cdots\left(x-b_{d+\rho(d)}\right),
\end{aligned}
$$

where $\phi, \rho \in S_{d}$. Let $f_{s}\left(x, h, c_{1}, \ldots, c_{d}\right)$ be a vector consisting of all unique polynomials in the nc variables $x, h$ and commuting variables $c=\left(c_{1}, \ldots, c_{d}\right)$ of the form

$$
\left(x-c_{\rho(1)}\right) \cdots\left(x-c_{\rho(s)}\right) h\left(x-c_{\rho(s+1)}\right) \cdots\left(x-c_{\rho(d)}\right),
$$

where $\rho \in S_{d}$. Note that the length of $f_{s}$ is $\left(\begin{array}{l}d \\ s\end{array}\right)$. Also, let $\mathbf{1}_{r, s}$ denote the $\left(\begin{array}{l}d \\ r\end{array}\right) \times\left(\begin{array}{l}d \\ s\end{array}\right)$ matrix all of whose entries are 1 . Then for all $\ell, r, s$,

$$
\begin{gathered}
\frac{1}{\ell !(k-\ell) !} \sum_{\tau \in S_{k}}\left(x-a_{\tau(1)}\right) \cdots\left(x-a_{\tau(\ell)}\right) f_{r}\left(x, h_{1}, b_{1}, \ldots, b_{d}\right)^{T}\left(x-a_{\tau(\ell+1)}\right) \\
\cdots\left(x-a_{\tau(d)}\right) \mathbf{1}_{r, s} f_{s}\left(x, h_{2}, b_{d+1}, \ldots, b_{2 d}\right)
\end{gathered}
$$

is equivalent to the sum of all distinct polynomials of the form (3.7), each of which has the coefficient

$$
\frac{1}{\left(\begin{array}{c}
2 d+k \\
\ell+2 d-(r+s)
\end{array}\right)(2 d+k+1)}
$$

in $\operatorname{Sym}\left(x-a_{1}, \ldots, x-a_{k}, x-b_{1}, \ldots, x-b_{2 d}, h_{1}, h_{2}\right)$.

Let

$$
W\left(x, h, c_{1}, \ldots, c_{d}\right)=\left(\begin{array}{c}
f_{0}\left(x, h, c_{1}, \ldots, c_{d}\right) \\
\vdots \\
f_{d}\left(x, h, c_{1}, \ldots, c_{d}\right)
\end{array}\right)
$$

let

and define

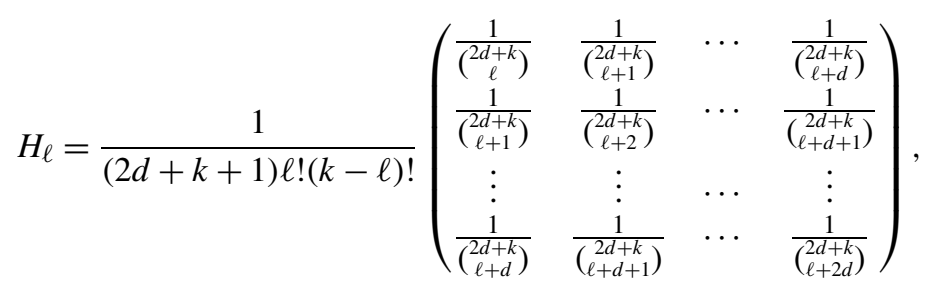

$$
C_{\ell}=\left(\left(H_{\ell}\right)_{r, s} \mathbf{1}_{r, s}\right) .
$$

By Lemma $3.7, H_{\ell}$ is positive semidefinite, and thus, by Lemma $3.8, C_{\ell}$ is positive semidefinite. Further, when we sum the polynomials (3.8) multiplied by coefficients (3.9) over all $(\ell, r, s)$, we get (3.4). 


\subsection{Connecting Trace-Convexity and Trace-Positivity}

A set $\mathcal{D} \subseteq \bigcup_{n} \mathbb{S}_{n}^{g}=: \mathbb{S}^{g}$ is called open if each $\mathcal{D}(n):=\mathcal{D} \cap \mathbb{S}_{n}^{g}$ is open.

Lemma 3.10. A symmetric nc polynomial $p \in \mathbb{R}\langle x\rangle$ is trace-convex on an open set $\mathcal{D} \subseteq \mathbb{S}^{g}$ if and only if its Hessian $p^{\prime \prime}(x)[h]$ is trace-positive on $\mathcal{D} \times \mathbb{S}^{g}$, that is,

$$
\operatorname{Tr} p^{\prime \prime}(X)[H] \geq 0
$$

for all $n \in \mathbb{N}, X \in \mathcal{D}(n)$ and $H \in \mathbb{S}_{n}^{g}$.

Proof. First, suppose that $p$ is trace-convex on $\mathcal{D}$. In this case, given $n \in \mathbb{N}, X \in$ $\mathcal{D}(n)$ and $H \in \mathbb{S}_{n}^{g}$, the polynomial of $t \in \mathbb{R}$,

$$
q(t)=\operatorname{Tr}(p(X+t H)+p(X-t H)-2 p(X))
$$

takes nonnegative values (for small enough $t$ ) and $q(0)=0$. Hence,

$$
0 \leq \frac{d^{2} q}{d t^{2}}(0)=\operatorname{Tr} p^{\prime \prime}(X)[H] .
$$

Thus, $p^{\prime \prime}$ is trace-positive on $\mathcal{D} \times \mathbb{S}^{g}$.

Now suppose $p^{\prime \prime}$ is trace-positive on $\mathcal{D} \times \mathbb{S}^{g}$ and let $n \in \mathbb{N}, X \in \mathcal{D}(n)$, and $H \in \mathbb{S}_{n}^{g}$ be given such that $X \pm H \in \mathcal{D}$. Consider the real polynomial

$$
r(t)=\operatorname{Tr}(p(X+t H)+p(X-t H)-2 p(X)) .
$$

Observe that $r(0)=0$,

$$
\frac{d r}{d t}(0)=\operatorname{Tr}\left(p^{\prime}(X)[H]-p^{\prime}(X)[H]\right)=0,
$$

and

$$
\frac{d^{2} r}{d t^{2}}(c)=\operatorname{Tr}\left(p^{\prime \prime}(X+c H)[H]+p^{\prime \prime}(X-c H)[H]\right) .
$$

In particular, by hypothesis the second (ordinary) derivative of $r$ is nonnegative. By Taylor's theorem, there is a $1>c>0$ such that

$$
\operatorname{Tr}(p(X+H)+p(X-H)-2 p(X))=r(1)=\frac{1}{2} \frac{d^{2} r}{d t^{2}}(c) \geq 0,
$$

and thus $p$ is trace-convex on $\mathcal{D}$.

The parallel between trace-convexity and matrix-convexity stops here due to the failure of a tracial version of Helton's sum of squares theorem [Hel02; McC01]. That is, a trace-positive nc polynomial is not necessarily a sum of Hermitian squares and commutators [KS08]. For more on matrix-positive polynomials, see, for example, [Hel02; HM04b; PNA10], and for trace-positive nc polynomials, we refer to [BK12; BCKP13] (see also [CDT10]) and the references therein. 


\subsection{Proof of the Main Results}

Proof of Theorem 3.1. Suppose (i) holds. Then $\frac{d^{2} p}{d x^{2}}$ is nonnegative on $\mathbb{R}$, which implies that it is a sum of polynomials of the form $A\left(x-b_{1}\right)^{2} \ldots\left(x-b_{d}\right)^{2}$ with $A \geq 0$. Lemma 3.6 and Proposition 3.9 now imply that $p^{\prime \prime}(x)[h]$ is a sum of squares plus commutators, that is, (iii) holds.

If (iii) holds, that is, $p^{\prime \prime}(x)[h]$ is a sum of Hermitian squares plus commutators, then it is clear that $\operatorname{Tr}\left(p^{\prime \prime}(x)[h]\right)$ is nonnegative (that is, $p^{\prime \prime}$ is trace positive), which implies, by Lemma 3.10, that $\operatorname{Tr}(p(x))$ is convex, establishing (ii). Finally, that (ii) implies (i) is obvious.

Proof of Theorem 3.2. (1) If $p^{\prime \prime}(x)[h]$ is of the form (3.1), then it is clear that for $a I \prec X \prec b I$ and all $H, \operatorname{Tr} p^{\prime \prime}(X)[H]$ is nonnegative. (Note that $(X-a I)(b I-$ $X) \succ 0$ since $X-a I$ and $b I-X$ commute.) So, by Lemma 3.10, $\operatorname{Tr} p$ is convex on $a I \prec X \prec b I$, and $p$ is convex on $(a, b)$.

Conversely, if $p$ is convex on $(a, b)$, then $\frac{d^{2} p}{d x^{2}}(x)$ is nonnegative for $x \in(a, b)$, which implies that it is a sum of polynomials each of one of the following forms [PR00]:

(a) $A\left(x-b_{1}\right)^{2} \cdots\left(x-b_{d}\right)^{2}$,

(b) $A(x-a)\left(x-b_{1}\right)^{2} \cdots\left(x-b_{d}\right)^{2}$,

(c) $A(b-x)\left(x-b_{1}\right)^{2} \cdots\left(x-b_{d}\right)^{2}$,

(d) $A(x-a)(b-x)\left(x-b_{1}\right)^{2} \cdots\left(x-b_{d}\right)^{2}$,

where $A>0$ and $d \geq 0$. The result now follows from applying Lemma 3.6 and Proposition 3.9.

The proofs of (2) and (3) are similar and left as an exercise for the reader.

\section{Concluding Remarks}

\subsection{An Alternative Proof of the Equivalence between Trace-Convexity and Convexity}

Here we present an alternative proof (cf. [Gui09, p. 74]) of the equivalence between trace-convexity and convexity for univariate nc polynomials, which avoids the sum of squares certificates. The argument applies naturally to continuous functions $p:(a, b) \rightarrow \mathbb{R}$.

Proposition 4.1. Let $a, b \in[-\infty, \infty]$ with $a<b$. If $p:(a, b) \rightarrow \mathbb{R}$ is continuous, then $\operatorname{Tr} p$ is convex on $a I \prec X \prec b I$ if and only if $p$ is convex on $(a, b)$.

Proof. If $\operatorname{Tr} p$ is convex on $(a, b)$, then for $1 \times 1$ matrices between $a I$ and $b I$, the trace $\operatorname{Tr} p$ is equal to $p$, and hence $p$ is convex on $(a, b)$.

For the converse, fix $t \in[0,1]$ and $X, Y \in \mathbb{S}_{n}$ for some $n \in \mathbb{N}$ such that $a I \prec$ $X, Y \prec b I$. Then $t X+(1-t) Y$ is a symmetric matrix and can be decomposed as $O^{T} \Lambda O$, where $O$ is orthogonal, and $\Lambda$ is diagonal with entries $\lambda_{1}, \ldots, \lambda_{n}$. Further,

$$
\left(O^{T} \Lambda O\right)^{n}=O^{T} \Lambda^{n} O \quad \text { and } \quad \operatorname{Tr} O^{T} \Lambda^{n} O=\operatorname{Tr} O O^{T} \Lambda^{n}=\operatorname{Tr} \Lambda^{n} .
$$


Extending this to general polynomials, we have

$$
\operatorname{Tr} p\left(O^{T} \Lambda O\right)=\operatorname{Tr} p(\Lambda)=\sum_{i=1}^{n} p\left(\lambda_{i}\right) .
$$

Conjugating by $O$ gives

$$
\Lambda=t O X O^{T}+(1-t) O Y O^{T} .
$$

Since both $X$ and $Y$ are symmetric, they can be decomposed as $X=O_{X}^{T} \Lambda_{X} O_{X}$ and $Y=O_{Y}^{T} \Lambda_{Y} O_{Y}$, where $O_{X}$ and $O_{Y}$ are orthogonal, and $\Lambda_{X}$ and $\Lambda_{Y}$ are diagonal with entries $\lambda_{i}^{X}$ and $\lambda_{i}^{Y}$, respectively.

Let $O^{T} O_{X}=A=\left(a_{i j}\right)_{1 \leq i, j \leq n}$ and $O^{T} O_{Y}=B=\left(b_{i j}\right)_{1 \leq i, j \leq n}$, which are both orthogonal matrices. The $i$ ith entry of the matrix $t O x O^{T}=t A^{T} \Lambda_{X} A$ is

$$
\left(t A^{T} \Lambda_{x} A\right)_{i i}=\sum_{k=1}^{n} t a_{i k}^{2} \lambda_{k}^{X} .
$$

Similarly, the $i$ ith entry of $(1-t) B^{T} \Lambda_{Y} B$ is

$$
\left((1-t) B^{T} \Lambda_{y}\right)_{i i}=\sum_{k=1}^{n}(1-t) b_{i k}^{2} \lambda_{k}^{Y} .
$$

Adding the two together gives

$$
\lambda_{i}=\sum_{k=1}^{n} t a_{i k}^{2} \lambda_{k}^{X}+\sum_{k=1}^{n}(1-t) b_{i k}^{2} \lambda_{k}^{Y} .
$$

Since $A$ and $B$ are orthogonal, $\sum_{k=1}^{n} a_{i k}^{2}=\sum_{k=1}^{n} b_{i k}^{2}=1$. Therefore, $\lambda_{i}$ is a convex combination of the $\lambda_{i}^{X}$ and $\lambda_{i}^{Y}$ terms. Further, since $a I \prec X, Y \prec b I$, each of the $\lambda_{i}^{X}$ and $\lambda_{i}^{Y}$ is in $(a, b)$. Therefore, if $p$ is convex on $(a, b)$, then

$$
\begin{aligned}
\operatorname{Tr}(t X+(1-t) Y) & =\operatorname{Tr} p\left(t O X O^{T}+(1-t) O Y O^{T}\right) \\
& =\sum_{i=1}^{n} p\left(\sum_{k=1}^{n} t a_{i k}^{2} \lambda_{k}^{X}+\sum_{k=1}^{n}(1-t) b_{i k}^{2} \lambda_{k}^{Y}\right) \\
& \leq \sum_{i=1}^{n}\left(\sum_{k=1}^{n} t a_{i k}^{2} p\left(\lambda_{k}^{X}\right)+\sum_{k=1}^{n}(1-t) b_{i k}^{2} p\left(\lambda_{k}^{Y}\right)\right) \\
& =\sum_{k=1}^{n}\left(t\left(\sum_{i=1}^{n} a_{i k}^{2}\right) p\left(\lambda_{k}^{X}\right)+\left(\sum_{i=1}^{n} b_{i k}^{2}\right) p\left(\lambda_{k}^{Y}\right)\right) \\
& =t \sum_{k=1}^{n} p\left(\lambda_{k}^{X}\right)+(1-t) \sum_{k=1}^{n} p\left(\lambda_{k}^{Y}\right) \\
& =t \operatorname{Tr} p(X)+(1-t) \operatorname{Tr} p(Y) .
\end{aligned}
$$

Hence, $\operatorname{Tr} p(X)$ is convex on $a I \prec X \prec b I$. 


\subsection{Algorithmic Aspects}

There are now several computer algebra packages available capable of assisting work in free convexity and free real algebraic geometry. Namely

(1) NCAlgebra [HOSM+] running under Mathematica;

(2) NCSOStools [CKP11] running under MATLAB.

The former is more universal in that it implements manipulation with noncommutative variables, including nc rationals and several algorithms pertaining to convexity. The latter is focused on free positivity, sums of squares and numerics.

EXAMPLE 4.2. Here is a simple example computed with the aid of NCSOStools. We demonstrate our results on $p=15 x^{2}-5 x^{4}+x^{6}$. Note that $\frac{d^{2} p}{d x^{2}}=(x-1)^{2} \times$ $(x+1)^{2}$, so $p$ is convex. To compute a noncommutative trace-convexity certificate, we proceed as follows:

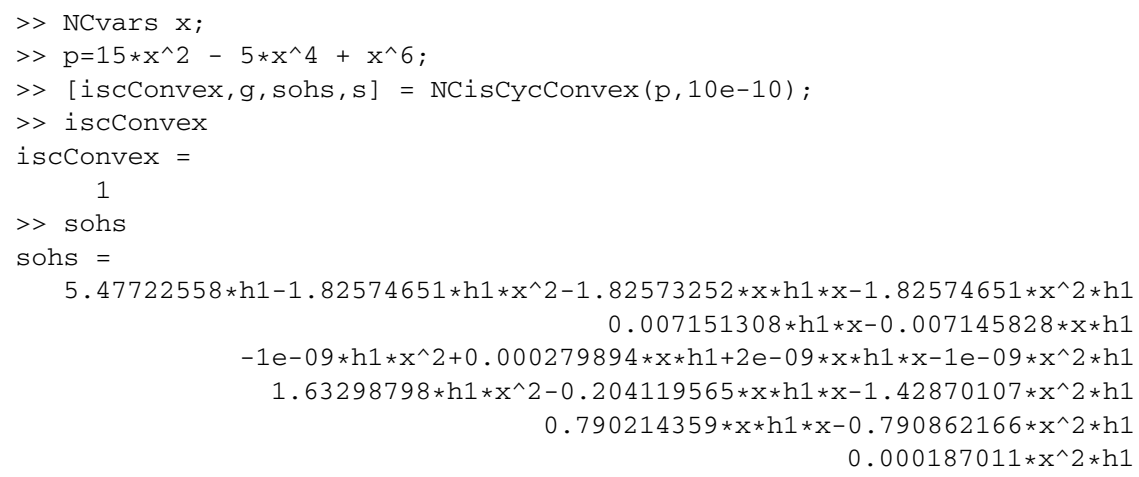

Since iscConvex $=1$, we believe that $p$ is trace-convex. To obtain an exact (symbolic) proof as opposed to the numerical evidence presented before, we proceed as follows. We try to manually find a sum of squares and commutators certificate of $p^{\prime \prime}(x)[h]$ as NCSOStoOIs then outputs more intermediate results that we can analyze.

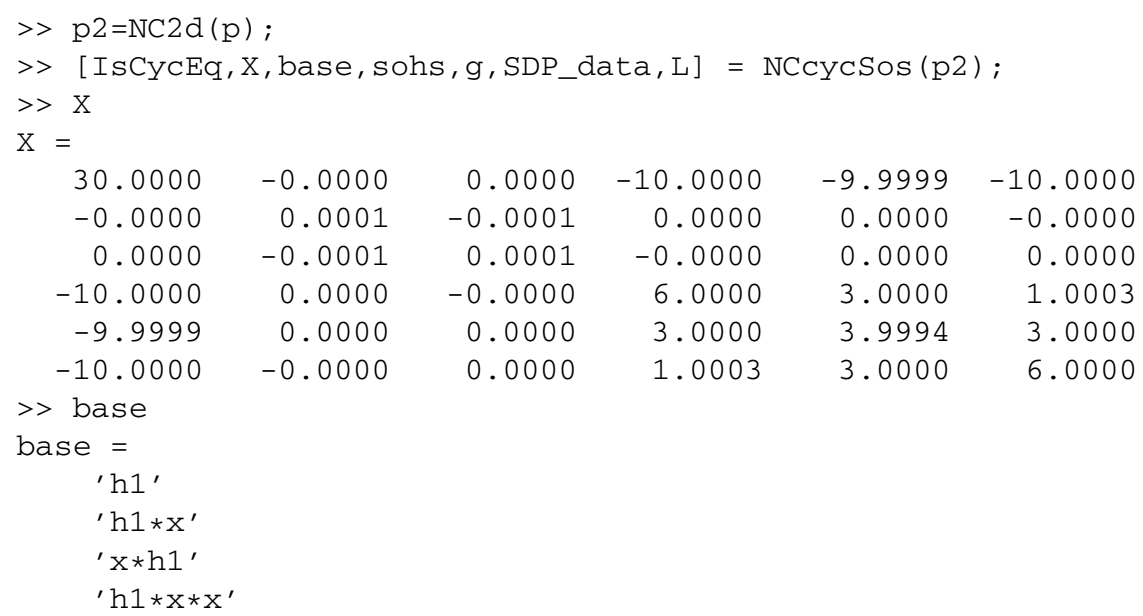




\footnotetext{
$' \mathrm{x} * \mathrm{~h} 1 * \mathrm{x}{ }^{\prime}$

$' \mathrm{x} * \mathrm{x} * \mathrm{~h} 1$ '
}

Here $\mathrm{X}$ is the Gram matrix corresponding to our sum of squares SDP (semidefinite program), and base is the corresponding border vector. The second and third rows and column of $\mathrm{X}$ are a direct summand corresponding to a polynomial cyclically equivalent to 0 . Thus, with

$$
X=\left(\begin{array}{cccc}
30 & -10 & -10 & -10 \\
-10 & 6 & 3 & 1 \\
-10 & 3 & 4 & 3 \\
-10 & 1 & 3 & 6
\end{array}\right), \quad v=\left(\begin{array}{c}
h \\
h x^{2} \\
x h x \\
x^{2} h
\end{array}\right)
$$

it is easy to verify

$$
v^{T} X v \stackrel{\text { cyc }}{\sim} p^{\prime \prime}
$$

and that $X \succeq 0$. Factor $X=R^{T} R$, where, with $s=\frac{1}{\sqrt{6}}, t=\sqrt{\frac{5}{2}}, u=\frac{\sqrt{30}}{3}$,

$$
R=\left(\begin{array}{cccc}
0 & 0 & 0 & 0 \\
0 & s & -2 s & s \\
0 & -t & 0 & t \\
-3 u & u & u & u
\end{array}\right)
$$

Letting $u=R v$, the three nonzero entries of $u$,

$$
\begin{aligned}
& q_{1}=s\left(h x^{2}-2 x h x+x^{2} h\right), \\
& q_{2}=t\left(h x^{2}-x^{2} h\right), \\
& q_{3}=u\left(3 h-h x^{2}-x h x-x^{2} h\right),
\end{aligned}
$$

satisfy

$$
p^{\prime \prime}(x)[h] \stackrel{\text { cyc }}{\sim} q_{1}^{*} q_{1}+q_{2}^{*} q_{2}+q_{3}^{*} q_{3} .
$$

\section{References}

[AM15] J. Agler and J. E. McCarthy, Pick interpolation for free holomorphic functions, Structured noncommutative multidimensional linear systems, Amer. J. Math. 137 (2015), 1685-1701.

[BGM05] J. A. Ball, G. Groenewald, and T. Malakorn, Structured noncommutative multidimensional linear systems, SIAM J. Control Optim. 44 (2005), 1474-1528.

[BGM06] _ Conservative structured noncommutative multidimensional linear systems, The state space method generalizations and applications, Oper. Theory Adv. Appl., 161, pp. 179-223, Birkhäuser, Basel, 2006.

[BCKP13] S. Burgdorf, K. Cafuta, I. Klep, and J. Povh, The tracial moment problem and trace-optimization of polynomials, Math. Program. 137 (2013), 557-578.

[BK12] S. Burgdorf and I. Klep, The truncated tracial moment problem, J. Operator Theory 68 (2012), 141-163.

[CKP11] K. Cafuta, I. Klep, and J. Povh, NCSOStools: a computer algebra system for symbolic and numerical computation with noncommutative polynomials, Optim. Methods Softw. 26 (2011), 363-380, Available from $\langle$ http://ncsostools.fis.unm.si〉. 
[CHSY03] J. F. Camino, J. W. Helton, R. E. Skelton, and J. Ye, Matrix inequalities: a symbolic procedure to determine convexity automatically, Integral Equations Operator Theory 46 (2003), 399-454.

[Car10] E. Carlen, Trace inequalities and quantum entropy: an introductory course, Entropy and the quantum, Contemp. Math., 529, pp. 73-140, Amer. Math. Soc., Providence, RI, 2010.

[CDT10] B. Collins, K. J. Dykema, and F. Torres-Ayala, Sum-of-squares results for polynomials related to the Bessis-Moussa-Villani conjecture, J. Stat. Phys. 139 (2010), 779-799.

[Dym07] H. Dym, Linear algebra in action, Grad. Stud. Math., 78, Amer. Math. Soc., Providence, RI, 2007.

[Eff09] E. G. Effros, A matrix convexity approach to some celebrated quantum inequalities, Proc. Natl. Acad. Sci. USA 106 (2009), 1006-1008.

[Gui06] A. Guionnet, Random matrices and enumeration of maps, International congress of mathematicians III, pp. 623-636, Eur. Math. Soc., Zürich, 2006.

[Gui09] L Large random matrices, Lecture Notes in Math., 1957, Springer, Berlin, 2009.

[GS09] A. Guionnet and D. Shlyakhtenko, Free diffusions and matrix models with strictly convex interaction, Geom. Funct. Anal. 18 (2009), 1875-1916.

[Han97] F. Hansen, Operator convex functions of several variables, Publ. Res. Inst. Math. Sci. 33 (1997), 443-463.

[Hel02] J. W. Helton, "Positive” noncommutative polynomials are sums of squares, Ann. of Math. (2) 156 (2002), 675-694.

[HM04a] J. W. Helton and S. McCullough, Convex noncommutative polynomials have degree two or less, SIAM J. Matrix Anal. Appl. 25 (2004), 1124-1139.

[HM04b] A Positivstellensatz for noncommutative polynomials, Trans. Amer. Math. Soc. 356 (2004), 3721-3737.

[HOSM+] J. W. Helton, M. C. de Oliveira, M. Stankus, and R. L. Miller, NCAlgebra, 2015, release edition. Available from 〈http://math.ucsd.edu/ ncalg〉

[KV14] D. S. Kaliuzhnyi-Verbovetskyi and V. Vinnikov, Foundations of free noncommutative function theory, Math. Surv. Monogr., 199, Amer. Math. Soc., Providence, RI, 2014.

[KS08] I. Klep and M. Schweighofer, Connes' embedding conjecture and sums of Hermitian squares, Adv. Math. 217 (2008), 1816-1837.

[Kra36] F. Kraus, Über konvexe Matrixfunktionen, Math. Z. 41 (1936), 18-42.

[McC01] S. McCullough, Factorization of operator-valued polynomials in several noncommuting variables, Linear Algebra Appl. 326 (2001), 193-203.

[OST07] H. Osaka, S. Silvestrov, and J. Tomiyama, Monotone operator functions, gaps and power moment problem, Math. Scand. 100 (2007), 161-183.

[PNA10] S. Pironio, M. Navascues, and A. Acin, Convergent relaxations of polynomial optimization problems with non-commuting variables, SIAM J. Optim. 20 (2010), 2157-2180.

[PR00] V. Powers and B. Reznick, Polynomials that are positive on an interval, Trans. Amer. Math. Soc. 352 (2000), 4677-4692.

[Pu13] M. Putinar, Sums of Hermitian squares: old and new (G. Blekherman, P. A. Parrilo, R. Thomas eds.), Semidefinite optimization and convex algebraic geometry, MOS-SIAM Ser. Optim., 13, pp. 407-469, SIAM, Philadelphia, PA, 2013. 
[SV06] D. Shlyakhtenko and D.-V. Voiculescu, Free analysis workshop summary: American Institute of Mathematics, 〈http://www.aimath.org/pastworkshops/ freeanalysis.html〉.

[Uci02] M. Uchiyama, Operator monotone functions and operator inequalities, Sugaku Expositions 18 (2005), 39-52.

\section{Klep}

Department of Mathematics

The University of Auckland

New Zealand

igor.klep@auckland.ac.nz

C. S. Nelson

Department of Mathematics

University of California

San Diego

csnelson@math.ucsd.edu
S. A. McCullough

Department of Mathematics

University of Florida

Gainesville

sam@math.ufl.edu 